\title{
Capecitabine and oxaliplatin combined with bevacizumab are feasible for treating selected Japanese patients at least 75 years of age with metastatic colorectal cancer
}

Yoshinori Munemoto ${ }^{1 \dagger}$, Mitsuro Kanda $^{2^{*}+}$, Keiichiro Ishibashi ${ }^{3}$, Taishi Hata ${ }^{4}$, Michiya Kobayashi $^{5}$, Junichi Hasegawa ${ }^{6}$, Mutsumi Fukunaga ${ }^{7}$, Akinori Takagane ${ }^{8}$, Toshio Otsuji ${ }^{9}$, Yasuhiro Miyake ${ }^{10}$, Michitaka Nagase ${ }^{11}$, Junichi Sakamoto ${ }^{12}$, Masaki Matsuoka ${ }^{13}$, Koji Oba $^{14,15}$ and Hideyuki Mishima ${ }^{16}$

\begin{abstract}
Background: Although number of elderly patients with metastatic colorectal cancer (mCRC) is rapidly increasing, this population is often underrepresented in clinical trials. Recently, a phase II trial demonstrated that capecitabine and oxaliplatin (XELOX) combined with bevacizumab XELOX plus bevacizumab was effective and well tolerated by elderly patients with $\mathrm{mCRC}$ who reside in Western countries. The aim of this study was to evaluate the safety and efficacy of XELOX plus bevacizumab for Japanese patients aged $\geq 75$ years with mCRC.

Methods: This prospective, open-label phase II trial recruited patients aged $\geq 75$ years with previously untreated mCRC between March 2010 and January 2012. Treatment consisted of $7.5 \mathrm{mg} / \mathrm{kg}$ of intravenous bevacizumab and $130 \mathrm{mg} / \mathrm{m}^{2}$ of oxaliplatin on day 1 of each cycle combined with $2000 \mathrm{mg} / \mathrm{m}^{2}$ of oral capecitabine per day on days 1-14 of each cycle. Treatment was repeated every 3 weeks until disease progression or termination of the study. The primary endpoint was progression-free survival; the secondary endpoints were toxicity, overall response rate, time-to-treatment failure, and overall survival.

Results: Thirty-six patients (male $58 \%$; median age 78 years; colon cancer $67 \%$ ) met all eligibility criteria and received at least one course of the planned treatment. The median time-to-treatment failure was 7.0 months. Twelve patients (33.3\%) experienced adverse effects (AEs) $\geq$ grade 3 and frequent AEs $\geq$ grade 3 , including neutropenia (22.2 \%) and neuropathy (13.9\%). Hypertension was the most frequent AE $\geq$ grade 3 associated with bevacizumab (11.1\%). Low baseline creatinine clearance associated significantly with the incidence of AEs $\geq$ grade 3. Response and disease control rates were 55.6 and $91.7 \%$, respectively. Median progression-free and overall survival times were 11.7 months (95\% confidence interval, 8.0-13.4 months) and 22.9 months, respectively.
\end{abstract}

Conclusion: XELOX combined with bevacizumab was well tolerated by selected Japanese patients aged $\geq 75$ years with $\mathrm{mCRC}$ patients, and controlled clinical trials are now required to determine the survival benefit.

Keywords: Colorectal cancer, Elderly, Bevacizumab, XELOX

\footnotetext{
* Correspondence: m-kanda@med.nagoya-u.ac.jp

${ }^{\dagger}$ Equal contributors

${ }^{2}$ Department of Gastroenterological Surgery (Surgery II), Nagoya University Graduate School of Medicine, 65 Tsurumai-cho, Showa-ku, Nagoya 466-8550, Japan

Full list of author information is available at the end of the article
}

C Biomed Central (c) 2015 Munemoto et al. Open Access This article is distributed under the terms of the Creative Commons Attribution 4.0 International License (http://creativecommons.org/licenses/by/4.0/), which permits unrestricted use, distribution, and reproduction in any medium, provided you give appropriate credit to the original author(s) and the source, provide a link to the Creative Commons license, and indicate if changes were made. The Creative Commons Public Domain Dedication waiver (http://creativecommons.org/publicdomain/zero/1.0/) applies to the data made available in this article, unless otherwise stated. 


\section{Background}

Colorectal cancer ranks worldwide as the third and fourth most common cancer in women and men, respectively, and the median survival of patients with metastatic colorectal cancer (mCRC) treated with best supportive care is approximately 6 months [1-3]. Treatment outcomes are improved considerably by newly developed chemotherapeutic agents and regimens. For example, treatment using 5-fluorouracil (5-FU) plus irinotecan, oxaliplatin, or both combined with targeted agents extends median overall survival (OS) to approximately 30 months $[4,5]$. Current guidelines recommend that first-line treatment for patients with mCRC should include doublet chemotherapy plus a targeted agent, if tolerated [6].

The prodrug capecitabine is activated by a unique mechanism that exploits the high activity of thymidine phosphorylase in malignant tissue that generates 5-FU preferentially in tumor tissue [7]. Capecitabine undergoes a three-step enzymatic conversion, and the final stage is catalyzed by thymidine phosphorylase, which is significantly more active in tumor tissue compared with healthy tissue $[7,8]$. Oral delivery of capecitabine simplifies chemotherapy and provides convenient outpatient therapy, because it avoids the complications and discomfort associated with intravenous administration and permits prompt discontinuation of treatment when toxicity occurs [9].

Combining capecitabine with oxaliplatin (XELOX) is advantageous for the reasons as follows: synergistic effects, no overlapping toxicities, easy to administer, and outpatient management [10-13]. Randomized phase III trials demonstrate that outcomes using first-line XELOX are comparable with those achieved using continuous infusion of 5-FU and folinic acid combined with oxaliplatin (FOLFOX) $[14,15]$. Moreover, combined with bevacizumab, a recombinant humanized version of a mouse monoclonal antibody against human vascular endothelial growth factor, XELOX achieves significantly improved progression-free survival (PFS) compared with chemotherapy alone [16-18].

The average age of the population is steadily increasing in many developed countries, particularly because of improvements in public health, nutrition, disease prevention, early detection, and continued progress in medical research [19]. The increase in patients' ages presents the medical community with new challenges. For example, more than $30 \%$ of patients with newly diagnosed CRC are aged at least 75 years [20]. Since the progressive reduction of functional reserve that occurs in various organs with ageing might increase the susceptibility of the elderly to adverse effects, clinical trials for elderly patients with $\mathrm{mCRC}$ have been conducted and tolerability of UFT/leucovorin, XELOX, capecitabine plus bevacizumab and S-1 plus bevacizumab were evaluated [21-26]. Yet, the safety and efficacy of XELOX plus bevacizumab for elderly patients remains to be determined because earlier large clinical trials limited eligibility to individuals $<70$ or 75 years owing to frail health $[16,17,27]$. Recently, a phase II trial (BECOX study) found that XELOX combined with bevacizumab is effective and well tolerated by patients aged $\geq 70$ years with $\mathrm{mCRC}$ who reside in Spain [28]. However, insufficient evidence is available to establish the safety and benefit of XELOX plus bevacizumab for Japanese patients with mCRC included in this age group. Moreover, lack of robust evidence of the new treatment described above may subject patients of advanced age to more conservative and less effective treatments. For example, older patients are more likely to receive monotherapy instead of combination therapy that does not include agents that target specific molecules [29-31].

Therefore, the aim of the present study was to evaluate the feasibility of XELOX plus bevacizumab for selected Japanese patients with $\mathrm{mCRC}$ aged $\geq 75$ years.

\section{Methods}

\section{Patients and methods}

A single-arm multicenter phase II trial (ASCA trial, Avastin plus XELOX Strategy for elderly patients with metastatic colorectal cancer) was planned to evaluate the safety and efficacy of XELOX plus bevacizumab for patients with $\mathrm{mCRC} \geq 75$ years of age [32]. The scientific and ethical validity of the study protocol was reviewed and approved by an internal review board of each participating facility (the Institutional Review Board at Osaka National Hospital, Osaka City General hospital, Osaka Rosai Hospital, Kitakyushu General Hospital, Kinki University, Kochi University, Fukui-ken Saiseikai Hospital, Saitama Medical Center, Jichi Medical University, Izumisano Municipal Hospital, Sakai City Hospital, Toyonaka Municipal Hospital, Dongo Hospital, Nara Social Insurance Hospital, Hakodate Goryoukaku Hospital, Fukuiken Saiseikai Hospital, Minoh City Hospital and Mimihara General Hopital). Written informed consent was obtained from all patients before enrollment. This study was conducted in accordance with the Declaration of Helsinki (2008) and registered with the University Hospital Medical Information Network (UMIN) Clinical Trial Registry as UMIN000003500 (http://www.umin.ac.jp/ctr/ index.htm).

Patients from 18 institutes were included in this study if they met all eligibility criteria as follows: (1) written informed consent before treatment; (2) age $\geq 75$ years when informed consent was granted; (3) Eastern Cooperative Oncology Group (ECOG) Performance Status (PS) of 0 or 1 ; (4) life expectancy $>3$ months; (5) histologically confirmed colorectal adenocarcinoma; (6) measurable 
disease consistent with the Response Evaluation Criteria in Solid Tumors (RECIST) version 1.1; (7) no prior chemotherapy (adjuvant chemotherapy included fluorouracil and/or oxaliplatin was allowed, but the last course of adjuvant chemotherapy must have concluded more than six months prior to colorectal cancer recurrence); (8) adequate function of vital organs, including liver and kidney (total bilirubin $\leq 1.5$-times the institutional upper normal limit, aspartate aminotransferase and alanine aminotransferase $\leq 2.5$-times the institutional upper normal limit, and serum creatinine $\leq$ institutional upper normal limit or creatinine clearance $(\mathrm{CCr}$, calculated using the Cockcroft-Gault formula) $\geq 50 \mathrm{ml} / \mathrm{min}$ ); adequate bone marrow function (leucocyte count $\geq 3000 / \mathrm{mm}^{3}$, neutrophil count $\geq 1500 / \mathrm{mm}^{3}$, platelet count $\geq 100,000 / \mathrm{mm}^{3}$, and hemoglobin $\geq 9.0 \mathrm{~g} / \mathrm{dl}$ ).

Key exclusion criteria included uncontrolled pleural effusion or ascites, brain metastasis, presence of other active malignancies, present or past (within the past 1 year) clinically significant cerebrovascular disease or thromboembolism, surgery planned during the course of the trial, anticoagulant treatment, coagulation disorder, nephropathy requiring medication or transfusion, uncontrolled hypertension or diabetes mellitus, uncontrolled diarrhea, history of bevacizumab treatment, and inability to take drugs orally [32].

\section{Treatment}

Treatment consisted of intravenous administration of $7.5 \mathrm{mg} / \mathrm{kg}$ of bevacizumab and $130 \mathrm{mg} / \mathrm{m}^{2}$ of oxaliplatin on day 1 of each cycle combined with $2000 \mathrm{mg} / \mathrm{m}^{2}$ oral capecitabine per day on days 1-14 of each cycle [32]. The end of the protocol treatment period was not prescribed. Treatment was repeated every 3 weeks until disease progression or termination of the study. The study protocol had no provisions regarding the secondline treatment. When patients exhibited adverse effects (AEs), the dose of each drug was reduced as specified in the study protocol that provided detailed algorithms to manage drug-specific toxicities such as oxaliplatin-related neuropathy, capecitabine-related diarrhea, hand-foot syndrome, bevacizumab-related hypertension, bleeding, and thromboembolism as well as other treatment-related toxicities. The dose reduction or stopping criteria of drugs due to adverse events is defined based on the haematological toxicity (Grade 4 neutropenia, Grade 3 febrile neutropeni a or Grade 3 or more decrease in platelets) and Grade 3 non-haematological toxicity. Dose reduction due to adverse events was performed for each drug as specified in the study protocol, which provided detailed algorithms to manage drug-specific toxicities such as oxaliplatin-related neuropathy as follows; G1, continue administration; G2/3, until recovery to $G 1$ or less and resume oxaliplatin with the reduction dose (for the first time $100 \mathrm{mg} / \mathrm{m}^{2}$, for the second time $85 \mathrm{mg} / \mathrm{m}^{2}$ ); $\mathrm{G} 4$, discontinuation of oxaliplatin.

\section{Study parameters}

Screening and baseline evaluations included assessing ECOG PS and conducting blood tests and physical examinations. Baseline tumor status with prospective identification of index lesions that were followed over the course of the study, was assessed using computed tomography (CT) studies of the chest, abdominal, and pelvis as well as determination of serum tumor-marker levels (carcinoembryonic antigen and carbohydrate antigen 19-9). During treatment, tumor status was assessed at the completion of each 8 -week cycle. RECIST ver. 1.1 was used to evaluate responses and determine disease progression. Response rate assessment was done locally. Toxicities, graded according to the criteria of the National Cancer Institute Common Terminology for Adverse Events (version 4.0), were evaluated during the study period and for 28 days after the last dose administered during the study by conducting physical examinations and laboratory tests (hematology, chemistry and electrolytes, and urinalysis), and evaluating ECOG PS. Patients who discontinued the protocol treatment were followed every 2 months until death or loss to follow-up. Neurotoxicity was graded as follows: G1 (asymptomatic) loss of deep tendon reflexes or paresthesia, G2 (moderate symptoms) limiting instrumental activities of daily living, G3 (severe symptoms) limiting daily self-care activities; G4 (lifethreatening consequences) urgent intervention indicated, and G5 (death). Patients were questioned about their use of concomitant medication and AEs. Association between the incidence of AEs $\geq \mathrm{G} 3$ and baseline CCr, American Society of Anesthesiologists (ASA) score (comorbidity index), ASA Physical Status Classification System score, age, body mass index (BMI), and sex were evaluated as potential risk factors for severe AEs.

\section{Statistical analysis}

The primary objective of the ASCA study was to determine PFS. Secondary endpoints were toxicity, overall response rate, time to treatment failure (TTF), and OS. Assuming a threshold PFS of 6.5 months and an estimated median PFS of 10.5 months, and referring to data from previous clinical trials we determined that a significance level $=95 \%$, an $\alpha$-error $=0.05$, and 32 patients were required. Estimating a loss as high as $10 \%$ of the final subject population, 35 patients were required. The Kaplan-Meier method was used to estimate survival, and the Cox proportional hazards model was used to calculate confidence intervals (CI). PFS was defined as the interval from the time of enrolment to the date of the first documented disease progression or a patient's death from any cause. OS was defined as the date of 
enrolment until the date of death from any cause. TTF was defined as the time from randomization to discontinuing treatment for any reason, including disease progression, treatment toxicity, patient preference, or death. The goodness-of-fit for AEs $\geq$ grade 3 was assessed by calculating the area under the curve (AUC), and optimal cutoff values were determined using the Youden index. The $\chi^{2}$ test was used to compare the difference between the values of two patient groups. A statistically significant difference was defined as $P<0.05$.

\section{Results}

\section{Patient characteristics}

Thirty-seven patients treated between March 2010 and January 2012 at 18 institutes were screened and met all eligibility requirements. One patient withdrew from the study before receiving treatment. The 36 patients (male $58 \%$; median age 78 years; colon cancer $67 \%$ ) enrolled received at least one course of the planned treatment. Baseline patient characteristics are shown in Table 1.

\section{Safety and response to treatment}

Patients were treated with a median of five cycles of XELOX plus bevacizumab (range 1-17), and the median relative dose intensities during the initial protocol (XELOX plus bevacizumab) were 86,89 , and $100 \%$ for capecitabine, oxaliplatin, and bevacizumab, respectively. There were 14 patients who continued to receive the protocol treatment after withdrawal of oxaliplatin (capecitabine with bevacizumab for 12 and capecitabine alone for two patients). The median TTF was 7.0 months (95 \% CI 4.7-10.8 months) (Fig. 1a). The reasons for discontinuing treatment were disease progression $(n=14)$, AEs $(n=14)$, withdrawal $(n=6)$, and surgery for metastases $(n=2)$. AEs that prevented continuing were as follows: neutropenia $(n=3)$, thrombotic disease $(n=2)$, anorexia $(n=2)$, ileus $(n=2)$, heart failure $(n=1)$, handfoot syndrome $(n=1)$, cerebral bleeding $(n=1)$, neuropathy $(n=1)$, and fatigue $(n=1)$.

Treatment-related toxicities are listed in Table 2 . Thirty-four (94.4 \%), and 12 (33.3\%) patients experienced AEs or AEs $\geq$ grade 3, respectively, and one treatment-related death was caused by intracranial bleeding. The latter patient was a 77-year-old woman with liver and lung metastasis without serious comorbidities who received seven courses of protocol treatment (XELOX plus bevacizumab) using the regular dose. During the eighth course, she lost consciousness, was diagnosed with intracerebral bleeding according to the results of a CT scan, and chemotherapy was discontinued. Frequent adverse events (any grade) were as follows: neuropathy (83.3\%), anemia (80.5\%), thrombocytopenia (58.3\%), hand-foot syndrome (58.3\%), and neutropenia (55.6\%). Frequent $\mathrm{AEs} \geq$ grade 3 were neutropenia $(22.2 \%)$ and
Table 1 Baseline patient characteristics $(n=36)$

\begin{tabular}{|c|c|}
\hline Clinical characteristic & Number of patients (\%) \\
\hline \multicolumn{2}{|l|}{ Sex } \\
\hline Male & $21(58.3)$ \\
\hline Female & $15(41.7)$ \\
\hline \multicolumn{2}{|l|}{ Age (years) } \\
\hline Median (range) & $78(75-86)$ \\
\hline \multicolumn{2}{|c|}{ ECOG performance status } \\
\hline 0 & $30(83.3)$ \\
\hline 1 & $6(16.7)$ \\
\hline \multicolumn{2}{|l|}{ Primary sites } \\
\hline Colon & $24(66.7)$ \\
\hline Rectum & $12(33.3)$ \\
\hline \multicolumn{2}{|l|}{ Primary tumor resection } \\
\hline Performed & $23(63.9)$ \\
\hline Not performed & $13(36.1)$ \\
\hline \multicolumn{2}{|l|}{ Adjuvant chemotherapy } \\
\hline Performed & $9(25.0)$ \\
\hline Not performed & $27(75.0)$ \\
\hline \multicolumn{2}{|c|}{ Appearance of metastasis } \\
\hline Synchronous & $19(52.7)$ \\
\hline Metachronous & $17(47.3)$ \\
\hline \multicolumn{2}{|l|}{ Metastatic sites } \\
\hline Liver & $21(58.3)$ \\
\hline Lung & $13(36.1)$ \\
\hline Lymph nodes & $14(38.9)$ \\
\hline Peritoneum & $2(5.6)$ \\
\hline Other & $2(5.6)$ \\
\hline \multicolumn{2}{|c|}{ Number of metastatic sites } \\
\hline 1 & $23(63.9)$ \\
\hline 2 & $11(30.5)$ \\
\hline 3 & $2(5.6)$ \\
\hline \multicolumn{2}{|c|}{ Creatinine clearance (mL/min) } \\
\hline Median (range) & $60.8(32.6-84.6)$ \\
\hline
\end{tabular}

neuropathy (13.9 \%). Bevacizumab-related AEs, proteinuria $(36.1 \%)$, and hypertension (27.8\%), were frequently observed for all grades, and the most frequent $\geq$ grade- 3 event was hypertension (11.1\%).

We evaluated the association between $\mathrm{AEs} \geq \mathrm{G} 3$ and baseline patient conditions including $\mathrm{CCr}$, comorbidity index, ASA Physical Status Classification System score, age, BMI, and sex. These findings identified baseline $\mathrm{CCr}$ as a potential predictor of AEs $\geq$ grade 3 . The AUC value of baseline $\mathrm{CCr}=0.69$, and the optimal cutoff value for predicting AEs $\geq$ grade $3=64 \mathrm{ml} / \mathrm{min}$ (sensitivity $=0.91$, specificity $=0.50$ (Additional file 1: Figure S1a). Further, patients with baseline $\mathrm{CCr}<64 \mathrm{ml} / \mathrm{min}$ had a 


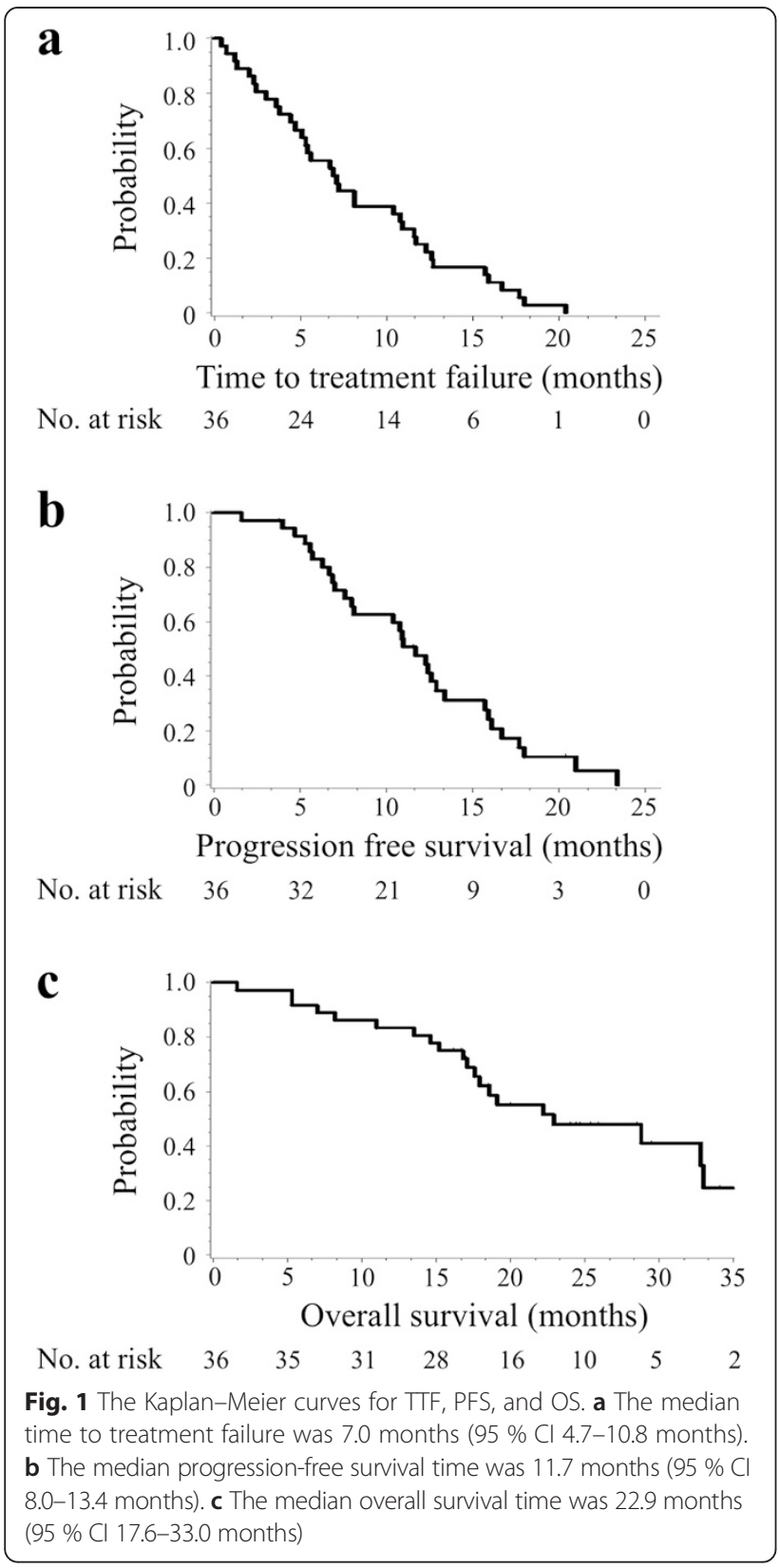

significantly higher incidence of AEs $\geq$ G3 compared with those with baseline $\mathrm{CCr} \geq 64 \mathrm{ml} / \mathrm{min}(77.8 \%$ and $22.2 \%$, respectively, $P=0.018$ ). No association was found between evaluated factors other than $\mathrm{CCr}$ (comorbidity index, ASA Physical Status Classification System score, age, BMI, and sex) and incidence of AEs $\geq$ G3.

\section{Efficacy}

The best radiographic response of each patient is presented in Additional file 1: Figure S1b. Responses to treatment were defined as follows: complete response $(\mathrm{CR})$, partial response (PR), stable disease (SD), and progressive disease, according to the RECIST ver. 1.1 definitions. The rates for CR, PR, and SD were 2.8, 52.8, and $36.1 \%$, respectively, and the response and disease control rates were 55.6 and $91.7 \%$, respectively (Table 3). The median PFS was 11.7 months (95 \% CI, 8.013.4 months (Fig. 1b), and the median OS was 22.9 months (95 \% CI 17.6-33.0 months, Fig. 1c).

\section{Discussion}

Robust evidence from the TREE 1 (XELOX) and TREE 2 (XELOX plus bevacizumab) (TREE1/2) randomized clinical trials shows that XELOX combined with bevacizumab offers survival benefits to patients with mCRC [33]. Unfortunately, insufficient evidence is available to insure the safety and benefits of combined treatment with XELOX and bevacizumab for patients aged $\geq 75$ years, that were often excluded from randomized trials, allegedly because of frail health or because they represented a minority of enrolled patients [16-18]. Feliu et al. conducted a recent phase II trial (BECOX study) in Spain and demonstrated that XELOX plus bevacizumab was effective and well tolerated by patients with mCRC aged $\geq 70$ years [28]. Here we designed a multicenter open-label phase II trial to evaluate the safety and efficacy of XELOX plus bevacizumab for Japanese patients aged $\geq 75$ years with $\mathrm{mCRC}$. The doses of capecitabine, oxaliplatin, and bevacizumab were determined with reference to the TREE1/2 trials [33], although the median age of patients enrolled in these studies was 62 years.

In the present study, we administered a median of five cycles of treatment (XELOX plus bevacizumab) (range, 1-17). Relative dose-intensities of capecitabine, oxaliplatin, and bevacizumab during the initial protocol (XELOX plus bevacizumab) were 86,89 , and $100 \%$, respectively. The median TTF was 7.0 months, although TTF represents a composite endpoint influenced by factors unrelated to efficacy, because discontinuation may be due to toxicity, patient preference, or a physician's reluctance to continue therapy. These results are similar to, or somewhat better compared with those of the TREE1/2 trials as well as those of the BECOX study [28, 33], despite the older patients studied here. The results of the present study and relevant clinical trials for $\mathrm{mCRC}$ were summarized in Table 4. Because therapeutic regimens with or without bevacizumab do not necessarily affect relative dose intensities of capecitabine and oxaliplatin, our results are comparable with the results of trials involving younger patients indicating that XELOX plus bevacizumab is well tolerated by patients aged $\geq 75$ years with mCRC.

The overall frequency of grade 3/4 AEs, including hematologic and nonhematologic events, is generally consistent with those of the TREE $1 / 2$ trials, an earlier phase I/II trial conducted in Japan and the BECOX study conducted in Spain [28, 33, 34]. The most characteristic finding here was that the incidence of grade- 1 neuropathy 
Table 2 Treatment-related adverse events

\begin{tabular}{|c|c|c|c|c|c|}
\hline & Grades 1/2 & Grade 3 & Grades 4/5 & All grades (\%) & $\geq$ Grade $3(\%)$ \\
\hline \multicolumn{6}{|l|}{ Hematologic AEs } \\
\hline Overall & & & & 94.4 & 33.3 \\
\hline Leucopenia & 14 & 1 & 0 & 41.7 & 2.8 \\
\hline Neutropenia & 12 & 7 & 1 & 55.6 & 22.2 \\
\hline Anemia & 27 & 2 & 0 & 80.5 & 5.6 \\
\hline Thrombocytopenia & 19 & 2 & 0 & 58.3 & 5.6 \\
\hline \multicolumn{6}{|l|}{ Non-hematologic AEs } \\
\hline Elevated AST & 11 & 0 & 0 & 30.5 & 0 \\
\hline Elevated ALT & 6 & 0 & 0 & 16.7 & 0 \\
\hline Hyperbilirubinemia & 2 & 0 & 0 & 5.6 & 0 \\
\hline Fatigue & 7 & 3 & 0 & 27.8 & 8.3 \\
\hline Anorexia & 9 & 2 & 0 & 27.8 & 5.6 \\
\hline Nausea & 4 & 0 & 0 & 11.1 & 0 \\
\hline Vomiting & 3 & 1 & 0 & 11.1 & 2.8 \\
\hline Diarrhea & 4 & 2 & 0 & 16.7 & 5.6 \\
\hline Stomatitis & 5 & 0 & 0 & 11.1 & 0 \\
\hline Hand-foot syndrome & 18 & 3 & 0 & 58.3 & 8.3 \\
\hline Injection site reaction & 3 & 0 & 0 & 8.3 & 0 \\
\hline Neuropathy (sensory) & 25 & 5 & 0 & 83.3 & 13.9 \\
\hline Allergy & 2 & 0 & 0 & 5.6 & 0 \\
\hline \multicolumn{6}{|c|}{ Bevacizumab-associated AEs } \\
\hline Hypertension & 6 & 4 & 0 & 27.8 & 11.1 \\
\hline Proteinuria & 11 & 2 & 0 & 36.1 & 5.6 \\
\hline Thrombosis & 0 & 1 & 0 & 2.8 & 2.8 \\
\hline Bleeding & 1 & 1 & 1 & 8.3 & 5.6 \\
\hline
\end{tabular}

$A E$ adverse effect

reached $83.3 \%$, and that of grades-3/4 neuropathy was $13.9 \%$. Cumulative neuropathy represents one of the major problems related to long-term therapy using oxaliplatin-containing regimens for patients with $\mathrm{mCRC}$, which is the main driver for trying to limit the dose of oxaliplatin $[12,14,28]$. The frequency (13.9\%) of G3/4 neuropathy encountered here was higher compared with those reported by earlier studies of Western cohorts

Table 3 Treatment profiles

\begin{tabular}{ll}
\hline Tumor response & $n(\%)$ \\
\hline CR & $1(2.8)$ \\
PR & $19(52.8)$ \\
SD & $13(36.1)$ \\
PD & $0(0)$ \\
Not evaluated & $3(8.3)$ \\
Response rate $(C R+P R)$ & $20(55.6)$ \\
Disease control rate $(C R+P R+S D)$ & $33(91.7)$
\end{tabular}

$C R$ complete response, $P R$ partial response, $S D$ stable disease, $P D$ progressive disease
(Table 4), although dose reduction and discontinuation of oxaliplatin was strictly defined in the study protocol $[13,28,35]$. A pilot study evaluating the safety of XELOX plus bevacizumab conducted in Japan reported a $17 \%$ frequency of neuropathy G3/4 $17 \%$, indicating that the frequency of severe neuropathy induced by XELOX plus bevacizumab differs between Western and Japanese patients [34]. Haller et al. showed the regional differences in tolerability of XELOX between the United States, East Asia, and the rest of the world [36]. Japanese patients experienced fewer G3/4 AEs during XELOX treatment compared with those from other regions, but no detailed data for neuropathy was provided.

Further, there remains room for discussion about the survival benefit of adding oxaliplatin. For example, in the AVEX study that evaluated capecitabine plus bevacizumab versus capecitabine alone in patients with $\mathrm{mCRC}$ aged $\geq 70$ years, the OS of those treated with capecitabine plus bevacizumab is similar OS to that our present study (20.7 months) [22]. Further, the FOCUS2 trial that compared capecitabine plus oxaliplatin with capecitabine 
Table 4 Summary of our results and relevant clinical trials for mCRC

\begin{tabular}{|c|c|c|c|c|c|c|c|c|c|c|c|}
\hline$\underline{\text { Study/first author }}$ & Phase & Treatment & Country & $\mathrm{n}$ & $\mathrm{ECOG}_{\mathrm{PS}}^{\mathrm{a}}$ & Age (median) & $\mathrm{RR}$ & PFS (month) & OS (month) & Neuropathy (G3/4) & Ref \\
\hline \multicolumn{12}{|l|}{ XELOX } \\
\hline Cassidy J & 2 & XELOX & 6 European, Canada & 96 & $0-1$ & $34-79(64)$ & $55 \%$ & 7.7 & 19.5 & $17 \%$ & 12 \\
\hline $\begin{array}{l}\text { TREE-1 } \\
\text { Hochster HS }\end{array}$ & 2 & mFOLFOX6 vs FOL vs XELOX & United States & 150 & $0-1$ & $31-84(62)$ & $\begin{array}{l}41 \% \text { vs } 20 \% \\
\text { vs } 27 \%\end{array}$ & $\begin{array}{l}8.7 \text { vs } 6.9 \\
\text { vs } 5.9\end{array}$ & $\begin{array}{l}19.2 \text { vs } 17.9 \\
\text { vs } 17.2\end{array}$ & $\begin{array}{l}18 \% \text { vs } 10 \% \\
\text { vs } 21 \%\end{array}$ & 33 \\
\hline Ducreux M & 3 & XELOX vs FOLFOX6 & France & 306 & $0-2$ & $32-84(65)$ & $42 \%$ vs $46 \%$ & 8.9 vs 9.3 & 20.1 vs 18.9 & $11.0 \%$ vs $25.5 \%$ & 15 \\
\hline \multicolumn{12}{|l|}{ BEV } \\
\hline $\begin{array}{l}\text { AVF2107g } \\
\text { Hurwitz H }\end{array}$ & 3 & IFL vs IFL + Bev & $\begin{array}{l}\text { United States, Australia, } \\
\text { New Zealand }\end{array}$ & 813 & $0-1$ & 18-(59) & $35 \%$ vs $45 \%$ & 6.2 vs 10.6 & 15.6 vs 20.3 & - & 17 \\
\hline $\begin{array}{l}\text { E3200 } \\
\text { Giantonio BJ }\end{array}$ & 3 & $\begin{array}{l}\text { FOLFOX4 vs FOLFOX4 + BEV vs } \\
\text { BEV }\end{array}$ & $\begin{array}{l}\text { United States, } \\
\text { South Africa }\end{array}$ & 829 & $0-2$ & $21-85(61)$ & $\begin{array}{l}8.6 \% \text { vs } 22.7 \% \\
\text { vs } 3.3 \%\end{array}$ & $\begin{array}{l}4.7 \text { vs } 7.3 \\
\text { vs } 2.7\end{array}$ & - & $\begin{array}{l}9.2 \% \text { vs } 16.3 \% \\
\text { vs } 0.8 \%\end{array}$ & 18 \\
\hline $\begin{array}{l}\text { FIRE-3 } \\
\text { Heinemann V }\end{array}$ & 3 & $\begin{array}{l}\text { FOLFIRI + cetuximab vs } \\
\text { FOLFIRI + BEV }\end{array}$ & Germany, Austria & 592 & $0-2$ & $27-79(65)$ & $62 \%$ vs $58 \%$ & 10.0 vs 10.3 & 28.7 vs 25.0 & $0.7 \%$ vs $1.4 \%$ & 4 \\
\hline $\begin{array}{l}\text { CALGB/SWOG } \\
80405^{b}\end{array}$ & 3 & $\begin{array}{l}\text { FOLFIRI or mFOLFOX } 6+\text { cetuximab } \\
\text { vs FOLFIRI or mFOLFOX } 6+\text { BEV }\end{array}$ & United States & 1137 & $0-1$ & $20-89(59)$ & - & 10.5 vs 10.8 & 29.9 vs 29.0 & $12 \%$ vs $14 \%$ & 5 \\
\hline \multicolumn{12}{|l|}{ XELOX + BEV } \\
\hline Wong NS & 2 & XELOX + BEV & United States & 50 & $0-2$ & 24-81 (55) & $50 \%$ & 10.3 & 23.3 & $14 \%$ & 11 \\
\hline $\begin{array}{l}\text { TREE-2 } \\
\text { Hochster HS }\end{array}$ & 2 & $\begin{array}{l}\text { mFOLFOX6 + BEV vs FOL + BEV vs } \\
\text { XELOX + BEV }\end{array}$ & United States & 223 & $0-1$ & $30-85(61)$ & $\begin{array}{l}52 \% \text { vs } 39 \% \\
\text { vs } 46 \%\end{array}$ & $\begin{array}{l}9.9 \text { vs } 8.3 \\
\text { vs } 10.3\end{array}$ & $\begin{array}{l}26.1 \text { vs } 20.4 \\
\text { vs } 24.6\end{array}$ & $\begin{array}{l}11 \% \text { vs } 9 \% \\
\text { vs } 11 \%\end{array}$ & 33 \\
\hline $\begin{array}{l}16966 \text { trial } \\
\text { Saltz LB }\end{array}$ & 3 & $\begin{array}{l}\text { FOLFOX4/XELOX vs FOLFOX4/ } \\
\text { XELOX + BEV }\end{array}$ & Worldwide & 1401 & $0-1$ & $18-86(60)$ & $38 \%$ vs $38 \%$ & 8.0 vs 9.4 & 19.9 vs 21.3 & - & 27 \\
\hline \multicolumn{12}{|l|}{ Elderly } \\
\hline $\begin{array}{l}\text { ASCA trial } \\
\text { Munemoto Y }\end{array}$ & 2 & XELOX + BEV & Japan & 36 & $0 .-1$ & $75-86(78)$ & $56 \%$ & 11.7 & 22.9 & $13.9 \%$ & - \\
\hline $\begin{array}{l}\text { SGOSG-CR0501 } \\
\text { Matsumoto T }\end{array}$ & 2 & $\mathrm{UFT}+\mathrm{LV}$ & Japan & 21 & $0-2$ & $75-83(79)$ & $33 \%$ & 5.3 & 18 & $0 \%$ & 21 \\
\hline Feliu J & 2 & Capecitabine + BEV & Spain & 59 & $0-2$ & $73-79(75)$ & $34 \%$ & 10.8 & 18.0 & $0 \%$ & 24 \\
\hline Feliu J & 2 & XELOX & Spain & 54 & $0-2$ & $70-82(76)$ & $36 \%$ & 5.8 & 13.2 & $2 \%$ & 25 \\
\hline $\begin{array}{l}\text { BECOX } \\
\text { Feliu J }\end{array}$ & 2 & XELOX + BEV & Spain & 69 & $0-1$ & $70-85$ (75) & $31 \%$ & 11.1 & 20.4 & $4 \%$ & 28 \\
\hline $\begin{array}{l}\text { BASIC trial } \\
\text { Yoshida M }\end{array}$ & 2 & $\mathrm{~S}-1+\mathrm{BEV}$ & Japan & 56 & $0-1$ & $66-85(75)$ & $57 \%$ & 9.9 & 25.0 & $0 \%$ & 26 \\
\hline $\begin{array}{l}\text { AVEX } \\
\text { Cunningham D }\end{array}$ & 3 & $\begin{array}{l}\text { Capecitabine vs capecitabine }+ \\
\text { BEV }\end{array}$ & Worldwide & 280 & $0-2$ & 70-87 (76) & $10 \%$ vs $19 \%$ & 5.1 vs 9.1 & 16.8 vs 20.7 & $0 \%$ & 22 \\
\hline $\begin{array}{l}\text { FOCUS2 } \\
\text { Seymour MT }\end{array}$ & 3 & $\begin{array}{l}\text { FL vs OxFU vs Capecitabine vs } \\
\text { XELOX }\end{array}$ & United Kingdom & 459 & $0-2$ & $35-87$ (74) & $\begin{array}{l}11 \% \text { vs } 38 \% \\
\text { vs } 14 \% \text { vs } 32 \%\end{array}$ & $\begin{array}{l}3.5 \text { vs } 5.8 \\
\text { vs } 5.2 \text { vs } 5.8\end{array}$ & $\begin{array}{l}10.1 \text { vs } 10.7 \\
\text { vs } 11.0 \text { vs } 12.4\end{array}$ & $\begin{array}{l}0 \% \text { vs } 1 \% \text { vs } 0 \% \\
\text { vs } 4 \%\end{array}$ & 23 \\
\hline
\end{tabular}

ECOG the Eastern Cooperative Oncology Group, PS performance status, RR response rate, PFS progression free survival, OS overall survival

aIn the eligibility criteria

${ }^{\mathrm{b}}$ Data from the 10th interim analysis (2014) 
alone, found no significant benefit of adding oxaliplatin [23]. Considering the high prevalence of neuropathy here, the benefit of adding oxaliplatin to capecitabine combined with bevacizumab for older Japanese patients with $\mathrm{mCRC}$ should be evaluated in clinical trials involving a large number of patients.

During the present study, one patient died because of treatment-related intracerebral bleeding. Although most AEs associated with bevacizumab (hypertension, proteinuria, and bleeding) are manageable, they infrequently lead to death. The patient had normal blood-clotting function as defined by the eligibility criteria, and the onset of intracerebral bleeding occurred after seven cycles of the protocol dose of XELOX plus bevacizumab. However, the overall safety profile of XELOX combined with bevacizumab for patients aged $\geq 75$ years was similar to those of previous clinical trials $[27,33]$. From our experience, we propose to monitor neurological signs on each visit and perform cerebral imaging on low threshold in symptomatic patients. In the present study, the incidence of AEs was independent of patients' sex, age, and BMI. In contrast, low baseline $\mathrm{CCr}(<64 \mathrm{ml} / \mathrm{min})$ was associated with the frequency of severe AEs, suggesting that baseline $\mathrm{CCr}$ should be considered as a determinant of the suitability of treating older patients with XELOX plus bevacizumab. However, further studies of a larger cohort are required.

Our trial achieved response and disease control rates of 55.6 and $91.7 \%$, respectively. The primary endpoint, median PFS, was 11.7 months (95 \% CI 8.0-13.4 months), and the median OS was 22.9 months (95\% CI 17.633.0 months). The median PFS in the TREE 2 trial, the earlier Japanese phase I/II trial, and the BECOX study were $10.3,11.0$ and 11.1 months, respectively [21, 28, 33]. The median OS of patients was 22.9 months in our present study, which is somewhat shorter compared with large studies of younger populations. For example, an OS of approximately 29 months was reported by the FIRE-3 and CALGB/SWOG 80405 trials $[4,5]$. In contrast, an earlier study of XELOX combined with bevacizumab for Western patients with mCRC aged 75 years demonstrated that OS was 20.4 months [28].

Folprecht et al. analyzed the differences in efficacy of 5 -FU-based chemotherapy between age groups $>70$ years and $<70$ years with mCRC, and concluded that elderly patients benefit at least to the same extent from palliative chemotherapy with 5-FU compared with younger patients [37]. Recently, Lieu et al. analyzed the large database of the ARCAD Clinical Trials Program and evaluated primary age effects and interactions with sex and PS [38]. They demonstrated that greater age was associated with poorer OS and PFS among treated patients with $\mathrm{mCRC}$ independent of sex and PS [38]. The main reason for the survival differences between our study and those of the FIRE-3 and CALGB/SWOG 80405 trials might be accounted for by the age of the patients rather than regional differences, and our results can be considered to reveal a reasonable outcome for patients aged $\geq 75$ years $[4,5]$.

The present study included some limitations as follows. The relatively small sample size precluded subgroup analysis of age, second-line treatment, and renal function. We selected patients according to strict eligibility criteria to ensure consistency with those of younger individuals. Therefore, these criteria may not be applicable to routine clinical practice. In addition, serial data were unavailable for blood cholesterol, triglyceride, and glucose concentrations that are influenced by capecitabine. The discussion might be limited due to lack of data on RAS/BRAF status. No elderly specific evaluation was conducted though the comprehensive geriatric assessment would have been of high value to learn about factors that are specific to the older patient population which could affect treatment outcome. Because the study protocol had no provisions regarding the second-line treatment, the detailed information of second-line treatment is unavailable. We were unable to determine the survival benefit of XELOX plus bevacizumab because this was a single-arm study.

\section{Conclusions}

Our results indicate that XELOX combined with bevacizumab was well tolerated by selected Japanese patients aged $\geq 75$ years with mCRC. Therefore, XELOX plus bevacizumab should not be withheld from these patients because of age alone. The survival benefit of this regimen must be determined by further controlled clinical trials.

\section{Additional file}

Additional file 1: Figure S1. Receiver operating characteristic curve and waterfall plot. (a) Receiver operating characteristic curve for baseline $\mathrm{CCr}$ as a predictor of AEs $\geq$ grade 3. The AUC and optimal cutoff values were 0.69 and $64 \mathrm{ml} / \mathrm{min}$, respectively. (b) Waterfall plot of maximum percentage tumor shrinkage. Progressive disease was not detected, and lesions with shrinkage of $\geq 30 \%$ were present in 20 patients (55.6 \%). (TIFF $6032 \mathrm{~kb})$

\section{Abbreviations}

mCRC: Metastatic colorectal cancer; 5-FU: 5-fluorouracil; OS: Overall survival; XELOX: Capecitabine and oxaliplatin; FOLFOX: Fluorouracil, folinic acid and oxaliplatin; PFS: Progression-free survival; UMIN: University Hospital Medical Information Network; ECOG: Eastern Cooperative Oncology Group; PS: Performance status; RECIST: Response evaluation criteria in solid tumors; CCr: Creatinine clearance; AE: Adverse effect; CT: Computed tomography; ASA: American Society of Anesthesiologist; BMI: Body mass index; TTF: Time to treatment failure; Cl: Confidence interval; AUC: Area under the curve; CR: Complete response; PR: Partial response; SD: Stable disease.

\section{Competing interests}

Keiichiro Ishibashi received lecture fees from Chugai Pharmaceutical Co., Ltd., Takeda Pharmaceutical Company Limited, Bayer Yakuhin, Ltd., Merck Serono Co., Ltd., Taiho Pharmaceutical Co., Ltd., Astellas Pharma. Inc., and Mitsubishi Tanabe Pharma Corporation. Junichi Sakamoto advises Takeda Pharmaceutical Company Limited and received lecture fees from Tsumura Co., Ltd. Hideyuki Mishima received lecture fees from Chugai Pharmaceutical Co., Ltd. and research funding from Chugai Pharmaceutical Co., Ltd. and Yakult Co., Ltd. 


\section{Authors' contributions}

MY, IK, HT, KM, HJ, FM, TA, MY, and NM collected cases and clinical data. $\mathrm{MM}, \mathrm{OT}$, and $\mathrm{MH}$ conceived and designed the study. OK conducted statistical analyses. MK prepared the initial manuscript. JS supervised the project. All authors contributed to the final manuscript and read and approved the final manuscript.

\section{Acknowledgements}

This study was supported, in part, by the nonprofit organization Epidemiological and Clinical Research Information Network (ECRIN).

\section{Author details}

${ }^{1}$ Department of Surgery, Fukuiken Saiseikai Hospital, Fukui, Japan. ${ }^{2}$ Department of Gastroenterological Surgery (Surgery II), Nagoya University Graduate School of Medicine, 65 Tsurumai-cho, Showa-ku, Nagoya 466-8550, Japan. ${ }^{3}$ Department of Digestive Tract and General Surgery, Saitama Medical Center, Saitama Medical University, Kawagoe, Japan. ${ }^{4}$ Department of Gastroenterological Surgery, Osaka University Graduate School of Medicine, Osaka, Japan. ${ }^{5}$ Department of Human Health and Medical Sciences, Kochi Medical School, Kohasu, Japan. ${ }^{6}$ Department of Surgery, Osaka Rosai Hospital, Sakai, Japan. ${ }^{7}$ Department of Surgery, Hyogo Prefectural Nishinomiya Hospital, Nishinomiya, Japan. ${ }^{8}$ Surgical Division, Hakodate Goryoukaku Hospital, Hakodate, Japan. ${ }^{9}$ Department of Internal Medicine, Dongo Hospital, Yamatotakada, Nara, Japan. ${ }^{10}$ Department of Surgery, Minoh City Hospital Gastrointestinal Research Center, Minoh, Osaka, Japan. ${ }^{11}$ Department of Surgical Oncology, Gifu University Graduate School of Medicine, Gifu, Japan. ${ }^{12}$ Director, Tokai Central Hospital, Gifu, Japan. ${ }^{13}$ Matsuoka Clinic, Kitakatsuragi, Nara, Japan. ${ }^{14}$ Department of Biostatistics, School of Public Health, Tokyo University Graduate School of Medicine, Tokyo, Japan.

${ }^{15}$ Interfaculty Initiative in Information Studies, Tokyo University, Tokyo, Japan.

${ }^{16}$ Unit of Cancer Center, Aichi Medical University, Nagakute, Japan.

\section{Received: 22 January 2015 Accepted: 8 October 2015}

\section{Published online: 24 October 2015}

\section{References}

1. Jemal A, Bray F, Center MM, Ferlay J, Ward E, Forman D. Global cancer statistics. CA Cancer J Clin. 2011;61:69-90.

2. Gruenberger B, Tamandl D, Schueller J, Scheithauer W, Zielinski C, Herbst F et al. Bevacizumab, capecitabine, and oxaliplatin as neoadjuvant therapy for patients with potentially curable metastatic colorectal cancer. J Clin Oncol. 2008:26:1830-5.

3. Scheithauer W, Rosen H, Kornek GV, Sebesta C, Depisch D. Randomised comparison of combination chemotherapy plus supportive care with supportive care alone in patients with metastatic colorectal cancer. BMJ. 1993;306:752-5.

4. Heinemann V, von Weikersthal LF, Decker T, Kiani A, Vehling-Kaiser U, Al-Batran SE, et al. FOLFIRI plus cetuximab versus FOLFIRI plus bevacizumab as first-line treatment for patients with metastatic colorectal cancer (FIRE-3): a randomised, open-label, phase 3 trial. Lancet Oncol. 2014;15:1065-75.

5. Venook AND, Lenz H, Innocenti F, Mahoney M, O'Neil B, Shaw J, et al. CALGB/SWOG 80405: Phase III trial of irinotecan/5-FU/leucovorin (FOLFIRI) or oxaliplatin/5-FU/leucovorin (mFOLFOX6) with bevacizumab (BV) or cetuximab (CET) for patients (pts) with KRAS wild-type (wt) untreated metastatic adenocarcinoma of the colon or rectum (MCRC). J Clin Oncol. 2014;32:5s.

6. Kirstein MM, Lange A, Prenzler A, Manns MP, Kubicka S, Vogel A. Targeted therapies in metastatic colorectal cancer: a systematic review and assessment of currently available data. Oncologist. 2014;19:1156-68.

7. Miwa M, Ura M, Nishida M, Sawada N, Ishikawa T, Mori K, et al. Design of a novel oral fluoropyrimidine carbamate, capecitabine, which generates 5-fluorouracil selectively in tumours by enzymes concentrated in human liver and cancer tissue. Eur J Cancer. 1998:34:1274-81.

8. Schuller J, Cassidy J, Dumont E, Roos B, Durston S, Banken L, et al. Preferential activation of capecitabine in tumor following oral administration to colorectal cancer patients. Cancer Chemother Pharmacol. 2000;45:291-7.

9. Cassidy J, Twelves C, Van Cutsem E, Hoff P, Bajetta E, Boyer M, et al. First-line oral capecitabine therapy in metastatic colorectal cancer: a favorable safety profile compared with intravenous 5-fluorouracil/leucovorin. Ann Oncol. 2002;13:566-75.
10. Borner MM, Dietrich D, Stupp R, Morant R, Honegger $H$, Wernli M, et al. Phase II study of capecitabine and oxaliplatin in first- and second-line treatment of advanced or metastatic colorectal cancer. J Clin Oncol. 2002;20:1759-66.

11. Wong NS, Fernando NH, Bendell JC, Morse MA, Blobe GC, Honeycutt W, et al. A phase II study of oxaliplatin, dose-intense capecitabine, and high-dose bevacizumab in the treatment of metastatic colorectal cancer. Clin Colorectal Cancer. 2011;10:210-6.

12. Cassidy J, Tabernero J, Twelves C, Brunet R, Butts C, Conroy T, et al. XELOX (capecitabine plus oxaliplatin): active first-line therapy for patients with metastatic colorectal cancer. J Clin Oncol. 2004;22:2084-91.

13. Makatsoris T, Kalofonos HP, Aravantinos G, Papadimitriou C, Kastritis E, Rigatos SK, et al. A phase II study of capecitabine plus oxaliplatin (XELOX): a new first-line option in metastatic colorectal cancer. Int J Gastrointest Cancer. 2005:35:103-9.

14. Martoni AA, Pinto C, Di Fabio F, Lelli G, Rojas Llimpe FL, Gentile AL, et al. Capecitabine plus oxaliplatin (xelox) versus protracted 5 -fluorouracil venous infusion plus oxaliplatin (pvifox) as first-line treatment in advanced colorectal cancer: a GOAM phase II randomised study (FOCA trial). Eur J Cancer. 2006;42:3161-8.

15. Ducreux M, Bennouna J, Hebbar M, Ychou M, Lledo G, Conroy T, et al. Capecitabine plus oxaliplatin (XELOX) versus 5-fluorouracil/leucovorin plus oxaliplatin (FOLFOX-6) as first-line treatment for metastatic colorectal cancer. Int J Cancer. 2011;128:682-90.

16. Kabbinavar FF, Hambleton J, Mass RD, Hurwitz HI, Bergsland E, Sarkar S. Combined analysis of efficacy: the addition of bevacizumab to fluorouracil/ leucovorin improves survival for patients with metastatic colorectal cancer. J Clin Oncol. 2005;23:3706-12.

17. Hurwitz H, Fehrenbacher L, Novotny W, Cartwright T, Hainsworth J, Heim W, et al. Bevacizumab plus irinotecan, fluorouracil, and leucovorin for metastatic colorectal cancer. N Engl J Med. 2004;350:2335-42.

18. Giantonio BJ, Catalano PJ, Meropol NJ, O'Dwyer PJ, Mitchell EP, Alberts SR, et al. Bevacizumab in combination with oxaliplatin, fluorouracil, and leucovorin (FOLFOX4) for previously treated metastatic colorectal cancer: results from the Eastern Cooperative Oncology Group Study E3200. J Clin Oncol. 2007;25:1539-44.

19. Groves R. National bibliography of US government publications. Statistical Abstract of the United States: 2008-2011 Washington, DC: US Census Bureau; 2011. 2011 http://www.census.gov/en.html

20. Gallego R, Sanchez N, Maurel J. Chemotherapy for elderly patients with advanced colorectal carcinoma. Expert Rev Anticancer Ther. 2006;6:795-800.

21. Matsumoto T, Nishina T, Mizuta M, Tsuji A, Watanabe R, Takahashi I, et al. Phase II study of first-line chemotherapy with uracil-tegafur plus oral leucovorin in elderly ( $>/=75$ years) Japanese patients with metastatic colorectal cancer: SGOSG-CR0501 study. Int J Clin Oncol. 2014.

22. Cunningham D, Lang I, Marcuello E, Lorusso V, Ocvirk J, Shin DB, et al. Bevacizumab plus capecitabine versus capecitabine alone in elderly patients with previously untreated metastatic colorectal cancer (AVEX): an open-label, randomised phase 3 trial. Lancet Oncol. 2013;14:1077-85.

23. Seymour MT, Thompson LC, Wasan HS, Middleton G, Brewster AE, Shepherd SF, et al. Chemotherapy options in elderly and frail patients with metastatic colorectal cancer (MRC FOCUS2): an open-label, randomised factorial trial. Lancet. 2011;377:1749-59.

24. Feliu J, Safont MJ, Salud A, Losa F, Garcia-Giron C, Bosch C, et al. Capecitabine and bevacizumab as first-line treatment in elderly patients with metastatic colorectal cancer. Br J Cancer. 2010;102:1468-73.

25. Feliu J, Salud A, Escudero P, Lopez-Gomez L, Bolanos M, Galan A, et al. XELOX (capecitabine plus oxaliplatin) as first-line treatment for elderly patients over 70 years of age with advanced colorectal cancer. Br J Cancer. 2006;94:969-75.

26. Yoshida M, Muro K, Tsuji A, Hamamoto Y, Yoshino T, Yoshida K, et al. Combination chemotherapy with bevacizumab and S-1 for elderly patients with metastatic colorectal cancer (BASIC trial). Eur J Cancer. 2015;51:935-41.

27. Saltz LB, Clarke S, Diaz-Rubio E, Scheithauer W, Figer A, Wong R, et al. Bevacizumab in combination with oxaliplatin-based chemotherapy as first-line therapy in metastatic colorectal cancer: a randomized phase III study. J Clin Oncol. 2008;26:2013-9.

28. Feliu J, Salud A, Safont MJ, Garcia-Giron C, Aparicio J, Vera R, et al. First-line bevacizumab and capecitabine-oxaliplatin in elderly patients with mCRC: GEMCAD phase II BECOX study. Br J Cancer. 2014;111:241-8.

29. McKibbin T, Frei CR, Greene RE, Kwan P, Simon J, Koeller JM. Disparities in the use of chemotherapy and monoclonal antibody therapy for elderly 
advanced colorectal cancer patients in the community oncology setting. Oncologist. 2008;13:876-85.

30. Khattak MA, Townsend AR, Beeke C, Karapetis CS, Luke C, Padbury R, et al. Impact of age on choice of chemotherapy and outcome in advanced colorectal cancer. Eur J Cancer. 2012;48:1293-8.

31. Sorbye H, Pfeiffer P, Cavalli-Bjorkman N, Qvortrup C, Holsen MH, WentzelLarsen T, et al. Clinical trial enrollment, patient characteristics, and survival differences in prospectively registered metastatic colorectal cancer patients. Cancer. 2009;115:4679-87.

32. Oba K, Matsuoka M, Satoh T, Muro K, Oriuchi N, Sakamoto J, et al. Multicentre phase II study of XELOX with bevacizumab in late-stage elderly patients with unresectable advanced/recurrent colorectal cancer: an ASCA study. Jpn J Clin Oncol. 2011;41:134-8.

33. Hochster HS, Hart LL, Ramanathan RK, Childs BH, Hainsworth JD, Cohn AL, et al. Safety and efficacy of oxaliplatin and fluoropyrimidine regimens with or without bevacizumab as first-line treatment of metastatic colorectal cancer: results of the TREE Study. J Clin Oncol. 2008:26:3523-9.

34. Doi T, Boku N, Kato K, Komatsu Y, Yamaguchi K, Muro K, et al. Phase I/II study of capecitabine plus oxaliplatin (XELOX) plus bevacizumab as first-line therapy in Japanese patients with metastatic colorectal cancer. Jpn J Clin Oncol. 2010;40:913-20.

35. Falcone A, Ricci S, Brunetti I, Pfanner E, Allegrini G, Barbara C, et al. Phase II trial of infusional fluorouracil, leucovorin, oxaliplatin, and irinotecan (FOLFOXIRI) compared with infusional fluorouracil, leucovorin, and irinotecan (FOLFIRI) as first-line treatment for metastatic colorectal cancer: the Gruppo Oncologico Nord Ovest. J Clin Oncol. 2007;25:1670-6.

36. Haller DG, Cassidy J, Clarke SJ, Cunningham D, Van Cutsem E, Hoff PM, et al. Potential regional differences for the tolerability profiles of fluoropyrimidines. J Clin Oncol. 2008;26:2118-23.

37. Folprecht G, Cunningham D, Ross P, Glimelius B, Di Costanzo F, Wils J, et al. Efficacy of 5-fluorouracil-based chemotherapy in elderly patients with metastatic colorectal cancer: a pooled analysis of clinical trials. Ann Oncol. 2004;15:1330-8

38. Lieu CH, Renfro LA, de Gramont A, Meyers JP, Maughan TS, Seymour MT, et al. Association of age with survival in patients with metastatic colorectal cancer: analysis from the ARCAD Clinical Trials Program. J Clin Oncol. 2014;32:2975-84.

\section{Submit your next manuscript to BioMed Central and take full advantage of:}

- Convenient online submission

- Thorough peer review

- No space constraints or color figure charges

- Immediate publication on acceptance

- Inclusion in PubMed, CAS, Scopus and Google Scholar

- Research which is freely available for redistribution

Submit your manuscript at www.biomedcentral.com/submit 